\title{
Bounds for Eigenvalues of Some Differential Operators by the Rayleigh-Ritz Method
}

\author{
By Burton Wendroff
}

1. Introduction. In $1943 \mathrm{R}$. Courant [1] suggested a variation of the RayleighRitz method for eigenvalue problems. In the classical Rayleigh-Ritz method one finds the stationary values of the Rayleigh quotient $J(u)$ as $u$ varies over a finitedimensional subspace of the space of admissible functions. The subspace usually consists of analytic functions, for example, polynomials. Courant's idea, in essence, is to use nonanalytic functions. For example, if the problem is

$$
\begin{aligned}
y^{\prime \prime}+\lambda y & =0, \\
y(0) & =y(1)=0,
\end{aligned}
$$

we divide the interval $[0,1]$ into $n$ equal intervals of length $h=1 / n$, the subintervals being $\left[x_{i}, x_{i+1}\right]$, where $x_{0}=0, x_{i}=x_{i-1}+h$, for $i=1,2, \cdots, n$. Now, let $S$ be the class of functions which are continuous on $[0,1]$, linear on each subinterval and satisfy (2). Then if

$$
\Lambda_{1}=\min _{s} J(u)=\min _{s} \frac{\int_{0}^{1}\left(u^{\prime}\right)^{2} d x}{\int_{0}^{1}\left(u^{2}\right) d x},
$$

and if $\lambda_{1}$ is the lowest eigenvalue of (1) we have

$$
\lambda_{1} \leqq \Lambda_{1} .
$$

In general, if $\lambda_{i}$ are the eigenvalues of (1) arranged in increasing order and if $\Lambda_{i}$ are the similarly arranged stationary values of $J(u)$ as $u$ varies over $S$, then

$$
\lambda_{i} \leqq \Lambda_{i} .
$$

The $\Lambda_{i}$ are the eigenvalues of the finite-difference equation

$$
\begin{aligned}
u_{i+1}-2 u_{i}+u_{i-1}+\Lambda \frac{h^{2}}{6}\left(u_{i+1}+4 u_{i}+u_{i-1}\right)=0, & i=1,2, \cdots, n-1, \\
u_{0}=u_{n}=0 . &
\end{aligned}
$$

We shall show for two boundary-value problems that a lower bound for $\lambda_{i}$ can be determined once $\Lambda_{i}$ is known. This is done by finding positive numbers $A$ and $B$ such that

$$
\Lambda_{i} \leqq \frac{\lambda_{i}+A}{B},
$$

Received September 21, 1964. This work was performed while the author was Visiting Associate Professor in the Division of Applied Mathematics of Brown University, while on leave of absence from the Los Alamos Scientific Laboratory, and it was supported by Office of Naval Research-Contract NONR-562(36). 
whence

$$
B \Lambda_{i}-A \leqq \lambda_{i} .
$$

This method of obtaining a lower bound has been used very effectively with a different definition of $\Lambda_{i}$ by H. F. Weinberger [4] and B. E. Hubbard [3].

In the last part of the paper we indicate how one might obtain higher-order bounds.

2. The Sturm-Liouville Problem. The general Sturn-Liouville problem is

$$
\begin{aligned}
\left(v y^{\prime}\right)^{\prime}+(\lambda p-q) y & =0, \\
a_{1} y^{\prime}(0)-b_{1} y(0) & =0, \\
a_{2} y^{\prime}(L)+b_{2} y(L) & =0,
\end{aligned}
$$

with $v$ and $p$ positive, $q$ non-negative on $[0, L]$, and $v$ piecewise continuously differentiable, $p$ and $q$ piecewise continuous on $[0, L]$. Also, $a_{i} \geqq 0, b_{i} \geqq 0$. We may assume without loss of generality that $v(x)=1, L=1$. This problem has positive eigenvalues $\lambda_{1}<\lambda_{2}<\cdots$ and corresponding eigenfunctions $y_{1}, y_{2}, \cdots$, normalized so that $\int_{0}^{1} p y_{i}^{2} d x=1$, which are continuously differentiable and have a continuous second derivative at each point of continuity of $p$ and $q$.

It is well known that

$$
\int_{0}^{1} p y_{i} y_{j} d x=\delta_{i j}
$$

For any function $f$ let

$$
\langle f\rangle=f(1)-f(0) .
$$

Let $a_{1}, \cdots, a_{s}$ be real numbers such that $\sum_{i=1}^{s} a_{i}^{2}=1$. Let $y(x)=\sum_{i=1}^{s} a_{i} y_{i}(x)$. Then it is known that

$$
\int_{0}^{1}\left(y^{\prime}\right)^{2} d x+\int_{0}^{1} q y^{2} d x-\left\langle y^{\prime} y\right\rangle \leqq \lambda_{8}
$$

(see [3, equation 2.12]).

Note that $-\left\langle y^{\prime} y\right\rangle$ is non-negative for any $y(x)$ which satisfies (5) and (6).

Choose mesh points $0=x_{0}<x_{1}<\cdots<x_{n}=1$ such that any discontinuity of $p$ or $q$ coincides with some $x_{j}$, and let $S_{1}$ be the space of functions which are continuous on $[0,1]$, linear in each $\left[x_{i}, x_{i+1}\right]$, and satisfy (5) and (6). For any continuous and piecewise continuously differentiable function $w(x)$ which satisfies the boundary conditions, let

$$
N(w)=\int_{0}^{1}\left(w^{\prime}\right)^{2} d x-\left\langle w w^{\prime}\right\rangle
$$

The Rayleigh quotient is

$$
J(w)=\frac{N(w)+\int_{0}^{1} q w^{2} d x}{\int_{0}^{1} p w^{2} d x}
$$


Let $0<\Lambda_{1}<\Lambda_{2}<\cdots<\Lambda_{n-1}$ be the stationary values of $J(w)$ as $w$ varies over $S_{1}$. Then from the Courant maximum-minimum principle we have

$$
\lambda_{i} \leqq \Lambda_{i}, \quad i=1,2, \cdots, n-1 .
$$

On the other hand, if $w_{1}, \cdots, w_{s}$ are any linearly independent functions in $S_{1}$, and if $\sum_{i=1}^{i} a_{i}{ }^{2}=1$ and $w(x)=\sum_{i=1}^{i} a_{i} w_{i}(x)$, then

$$
\Lambda_{\mathbf{6}} \leqq \max _{a_{i}, \cdots, a_{\text {s }}} J(w) \text {. }
$$

Weinberger calls this the Poincare inequality, and it follows immediately from the minimum-maximum character of the $\Lambda_{i}$.

Now, let $Y_{i}(x)$ be that function in $S_{1}$ which agrees with the eigenfunction $y_{i}(x)$ at each interior $x_{j}$, i.e,

$$
Y_{i}\left(x_{j}\right)=y_{i}\left(x_{j}\right), \quad j=1,2, \cdots, n-1, \quad i=1,2, \cdots, n-1,
$$

and let

$$
Y(x)=\sum_{i=1}^{\dot{1}} a_{i} Y_{i}(x)
$$

We shall show later that if the intervals are sufficiently small the $Y_{i}$ are linearly independent. Then from (10) we have

$$
\Lambda_{\mathbf{8}} \leqq \max _{a_{i}, \cdots a_{0}} J(Y) .
$$

Let

$$
r(x)=y(x)-Y(x) .
$$

Then

$$
r\left(x_{j}\right)=0, \quad j=1,2, \cdots, n-1,
$$

and $r$ satisfies (5) and (6). In addition, $r(x)$ is twice continuously differentiable in each $\left(x_{j}, x_{j+1}\right)$, and $r^{\prime \prime}(x)=y^{\prime \prime}(x)$. Let $G_{i}(x, \xi)$ be the Green's function for the differential operator $d^{2} / d x^{2}$ on the interval $\left[x_{i}, x_{i+1}\right]$, with boundary conditions (12) or $(5)$ or $(6)$, whichever applies. Then

$$
r(x)=\int_{x_{j}}^{x_{j+1}} G_{j}(x, \xi) y^{\prime \prime}(\xi) d \xi, \quad x_{j} \leqq x \leqq x_{j+1}
$$

Let us first consider the denominator of $J(Y)$. We have

$$
Y^{2} \geqq y^{2}-2|y||r|,
$$

and

$$
\int_{x_{j}}^{x_{j+1}} p Y^{2} d x \geqq \int_{x_{j}}^{x_{j+1}} p y^{2} d x-2 \int_{x_{j}}^{x_{j+1}} p|y||r| d r
$$

Therefore,

$$
\int_{0}^{1} p Y^{2} d x \geqq 1-2 \sqrt{ } p_{M} \sum_{j=0}^{n-1} \int_{x_{j}}^{x_{j+1}} \sqrt{ } p|y||r| d x,
$$


where, for any $f(x)$,

$$
\begin{aligned}
f_{M} & =\max _{[0,1]} f(x), \\
f_{m} & =\min _{[0,1]} f(x) .
\end{aligned}
$$

By Schwartz' inequality,

$$
\left(\int_{x_{j}}^{x_{j+1}} \sqrt{ } p|y||r| d x\right)^{2} \leqq \int_{x_{j}}^{x_{j+1}} p y^{2} d x \int_{x_{j}}^{x_{j+1}} r^{2} d x
$$

But, from (13),

$$
r^{2}(x) \leqq \int_{x_{j}}^{x_{j+1}} G_{j}{ }^{2}(x, \xi) d \xi \int_{x_{j}}^{x_{j+1}}\left(y^{\prime \prime}\right)^{2} d \xi
$$

Put

$$
G=\max _{j} \int_{x_{j}}^{x_{j+1}} \int_{x_{j}}^{x_{j+1}} G_{j}^{2}(x, \xi) d x d \xi
$$

Then

$$
\int_{x_{j}}^{x_{j+1}} \sqrt{ } p|y||r| d x \leqq \sqrt{ } G\left[\int_{x_{j}}^{x_{j+1}} p y^{2} d x \int_{x_{j}}^{x_{j+1}}\left(y^{\prime \prime}\right)^{2} d x\right]^{1 / 2}
$$

and

$$
\sum_{j=0}^{n-1} \int_{x_{j}}^{x_{j+1}} \sqrt{ } p|y||r| d x \leqq \sqrt{ } G\left[\int_{0}^{1}\left(y^{\prime \prime}\right)^{2} d x\right]^{1 / 2} .
$$

From the differential equation,

$$
y^{\prime \prime}=\sum_{i=1}^{s} a_{i}\left(q-\lambda_{i} p\right) y_{i} .
$$

Then for any $\gamma>0$,

$$
\int_{0}^{1}\left(y^{\prime \prime}\right)^{2} d x \leqq(1+\gamma) \frac{q_{M}{ }^{2}}{p_{m}}+\left(1+\frac{1}{\gamma}\right) p_{M} \Lambda_{s}^{2} .
$$

Let

$$
K_{s}=\underset{\gamma>0}{\text { g.l.b. }}\left[(1+\gamma) \frac{q_{M}{ }^{2}}{p_{m}}+\left(1+\frac{1}{\gamma}\right) p_{M} \Lambda_{s}{ }^{2}\right]^{1 / 2},
$$

so that if $q_{M}=0, K_{s}=\Lambda_{s} \sqrt{ } p_{M}$, otherwise $\gamma=\Lambda_{s} \sqrt{ }\left(p_{M} p_{m}\right) / q_{M}$. In any event, from (15), (18) we have

$$
\int_{0}^{1} p Y^{2} d x \geqq 1-2 \sqrt{ } p_{M} K_{s} \sqrt{ } G .
$$

The important thing to note at this point is that $K_{\varepsilon}$ and $G$ depend only on the data. For example, if $q(x)=0, a_{1}=a_{2}=0$, then

$$
\int_{0}^{1} p Y^{2} d x \geqq 1-\frac{2 p_{M} \Lambda_{s}}{3 \sqrt{ } 10} \max _{j}\left|x_{j+1}-x_{j}\right|^{2} .
$$


The linear independence of the $Y_{i}$ is implied by (19), for $\max \left(x_{j+1}-x_{j}\right)$ sufficiently small.

By entirely similar methods we find

$$
\int_{0}^{1} q Y^{2} d x \leqq \int_{0}^{1} q y^{2} d x+\frac{2 q_{M}}{\sqrt{ } p_{m}} K_{8} \sqrt{ } G+q_{M} G K_{8}^{2}
$$

We now show that

$$
N(y)=N(Y)+N(r)
$$

From the identity

$$
-\int_{x_{j}}^{x_{j+1}} y^{\prime \prime} y d x=-\int_{x_{j}}^{x_{j+1}}\left(Y^{\prime \prime}+r^{\prime \prime}\right)(Y+r) d x
$$

follows the identity

$$
\begin{aligned}
\left.y^{\prime} y\right|_{j} ^{j+1}+\int_{x_{j}}^{x_{j+1}}\left(y^{\prime}\right)^{2} d x=-\left.y^{\prime} Y\right|_{j} ^{j+1}+\left.r Y^{\prime}\right|_{j} ^{j+1} & -\left.r^{\prime} r\right|_{j} ^{j+1} \\
& +\int_{x_{j}}^{x_{j+1}}\left(Y^{\prime}\right)^{2} d x+\int_{x_{j}}^{x_{j+1}}\left(r^{\prime}\right)^{2} d x .
\end{aligned}
$$

Noting that $y, y^{\prime}$ and $Y$ are continuous and $r\left(x_{j}\right)=0$ we obtain, by summing the above,

$$
\begin{aligned}
N(y) & =-\left\langle y^{\prime} Y\right\rangle+\left\langle r Y^{\prime}\right\rangle+\int_{0}^{1}\left(Y^{\prime}\right)^{2} d x+N(r) \\
& =-\left\langle y^{\prime} Y\right\rangle+\left\langle y Y^{\prime}\right\rangle-\left\langle Y Y^{\prime}\right\rangle+\int_{0}^{1}\left(Y^{\prime}\right)^{2} d x+N(r) \\
& =N(Y)+N(r) .
\end{aligned}
$$

But $N(r) \geqq 0$, so from (11), (8), (21) and (19), we obtain

$$
\Lambda_{s} \leqq \frac{\lambda_{s}+\frac{2 q_{M}}{p_{m}} K_{s} \sqrt{ } G+q_{M} K_{s}^{2} G}{1-2 \sqrt{ } p_{M} K_{s} \sqrt{ } G},
$$

which is the desired result.

It should be noted that there would have been no terms other than $\lambda_{\text {, }}$ in the numerator of (22) if we could have composed $S_{1}$ of solutions of $Y^{\prime \prime}=q Y$ rather than of $Y^{\prime \prime}=0$.

3. A Fourth-Order Problem. Certain fourth-order problems can be handled by the methods of Section 2. For example, consider the boundary-value problem

$$
\begin{aligned}
\left(v y^{\prime \prime}\right)^{\prime \prime} & =\lambda p y, \\
y(0) & =y(1)=y^{\prime}(0)=y^{\prime}(1)=0,
\end{aligned}
$$

which describes the fundamental modes of vibration of a clamped beam. The Rayleigh quotient is

$$
J(w)=\frac{\int_{0}^{1}\left(v w^{\prime \prime}\right)^{2} d x}{\int_{0}^{1} p w^{2} d x} .
$$


We define $\Lambda_{i}$ to be the stationary values of $J(w)$ as $w$ varies over a class of functions which we call $T_{3}$, which is composed of all functions which are continuously differentiable on $[0,1]$, satisfy $(24)$, and which in each interval $\left(x_{j+1}, x_{j}\right)$ satisfy $\left(v w^{\prime \prime}\right)^{\prime \prime}=0$. Any discontinuities of $v$ and $p$ must coincide with some $x_{j}$. The elements of $T_{3}$ have the following form: $w(x) \in T_{3}$ if and only if there exist real numbers $w_{j}, w_{j}^{\prime}, j=1,2, \cdots, n-1$, and constants $a_{j}, b_{j}, c_{j}, d_{j}$ such that

$$
w(x)=a_{j} \int_{x_{j}}^{x} \int_{x_{j}}^{t} \frac{s d s}{v(s)}+b_{j} \int_{x_{j}}^{x} \int_{x_{j}}^{t} \frac{d s}{v(s)}+c_{j}\left(x-x_{j}\right)+d_{j},
$$

for $x_{j} \leqq x \leqq x_{j+1}$, and $w\left(x_{j}\right)=w_{j}, w^{\prime}\left(x_{j}\right)=w_{j}^{\prime} . J$ is stationary at some $w(x)$ if

$$
\frac{\partial J}{\partial w_{j}}=0, \quad \frac{\partial J}{\partial w_{j}^{\prime}}=0, \quad j=1,2, \cdots, n-1 .
$$

As before we have $\lambda_{i} \leqq \Lambda_{i}$. To obtain an upper bound for $\Lambda_{i}$ we let $y_{i}$ be the normalized eigenfunctions of (23) and (24) and let $Y_{i}$ be the element in $T_{3}$ such that

$$
Y_{i}\left(x_{j}\right)=y_{i}\left(x_{j}\right), Y_{i}^{\prime}\left(x_{j}\right)=y_{i}^{\prime}\left(x_{i}\right) \text {, }
$$

$j=0,1, \cdots, n$. Then for $Y=\sum_{i=1}^{8} a_{i} Y_{i}, \sum_{i=1}^{8} a_{i}^{2}=1$,

$$
\Lambda_{s} \leqq \max _{a_{1}, \cdots, a_{s}} J(Y)
$$

If we replace $G_{i}(x, \xi)$ in (13) by the Green's function for the operator

$$
\frac{d^{2}}{d x^{2}} v \frac{d^{2}}{d x^{2}}
$$

with boundary conditions $r\left(x_{j}\right)=r^{\prime}\left(x_{j}\right)=0, j=0,1, \cdots, n$, then

$$
\int_{0}^{1} p Y^{2} d x \geqq 1-2 p_{M} \Lambda_{s} \sqrt{ } G \text {. }
$$

It is easily verified that

$$
\int_{0}^{1} v\left(Y^{\prime \prime}\right)^{2} d x \leqq \int_{0}^{1} v\left(y^{\prime \prime}\right)^{2} d x \leqq \lambda_{s},
$$

so

$$
\Lambda_{8} \leqq \frac{\lambda_{s}}{1-2 p_{M} \Lambda_{s} \sqrt{ } G}
$$

An interesting special case occurs when $v(x)$ is a step function. Then the elements of $T_{3}$ are cubic polynomials in each $\left[x_{j+1}, x_{j}\right]$, and

$$
\Lambda_{s} \leqq \frac{\lambda_{s}}{1-\left(\frac{p_{M}}{v_{m}}\right) 4.05 \times 10^{-3} \max \left[x_{j+1}-x_{j}\right]^{4}} .
$$

The matrix eigenvalue problem defining the $\lambda_{i}$ can be found in [5], where it is assumed that both $v$ and $p$ are step functions and that the beam is simply supported. The matrices for the clamped beam are found by discarding the first and last intervals. 
4. Higher-Order Bounds. To attempt to obtain higher-order bounds for the Sturm-Liouville problem, rather than use higher-order Lagrange interpolation, as is done in [2], we replace $S_{1}$ by the space $S_{2 k+1}, k=1,2, \cdots$, consisting of all functions satisfying (5) and (6) which are $k$ times continuously differentiable on $[0,1]$ and which are polynomials of degree $2 k+1$ in each $\left[x_{j+1}, x_{j}\right]$. If the eigenfunctions are sufficiently smooth, there will be functions $Y_{i} \in S_{2 k+1}$ such that

$$
Y_{i}^{(\alpha)}\left(x_{j}\right)=y_{i}^{(\alpha)}\left(x_{j}\right), \quad \alpha=0,1, \cdots, k .
$$

With $y=Y+r$ we have

$$
r(x)^{(2 k+2)}=y(x)^{(2 k+2)} .
$$

with appropriate boundary conditions, so that there are Green's functions $G_{j}(x, \xi)$ such that

$$
r(x)=\int_{x_{j}}^{x_{j+1}} G_{j}(x, \xi) y(\xi)^{(2 k+2)} d \xi .
$$

We proceed as before; however, estimating $\int_{0}^{1}\left[y^{(2 k+2)}\right]^{2} d x$ will be quite difficult for $k>0$ unless $p(x)$ and $q(x)$ are step functions, in which case we would have $y_{i}^{(2 h+3)}=$ $\left(q-\lambda_{i} p\right)^{k+1} y_{i}$, from which estimates can be made.

For the numerator of the Rayleigh quotient we no longer have $N(Y) \leqq N(y)$, but

$$
\begin{aligned}
N(Y) & \leqq N(y)+2 \int_{0}^{1}\left|Y^{\prime \prime} r\right| d x \\
& \leqq N(y)+2 \int_{0}^{1}\left|y^{\prime \prime} r\right| d x+2 \int_{0}^{1}\left|r^{\prime \prime} r\right| d x,
\end{aligned}
$$

which can be estimated if $r$ can.

For the fourth-order problem we could use spaces $T_{2 k+1}$ consisting of functions satisfying the boundary conditions such that, in each subinterval,

$$
\left(v w^{\prime \prime}\right)^{\prime \prime}=\sum_{i=0}^{2 k-3} a_{i} x^{1}
$$

where the $2 k-2$ constants $a_{0}, \cdots, a_{2 k-3}$ and the four constants of integration are determined by the condition that $w^{(\alpha)}$ be continuous for $\alpha=0,1, k$. We have not obtained any bounds using $T_{2 k+1}$.

Los Alamos Scientific Laboratory

Los Alamos, New Mexico

1. R. Courant, "Variational methods for the solution of problems of equilibrium and vibrations," Bull. Amer. Math. Soc., v. 49, 1943, pp. 1-23. MR \$, 200.

2. C. C. Farrington, R. T. Gregory \& A. H. TAUB, "On the numerical solution of SturmLiouville differential equations," MTAC, v. 11, 1957, pp. 131-150. MR 19, 772.

3. B. E. Hubbard, "Bounds for eigenvalues of the Sturm-Liouville problem by finite difference methods," Arch. Rational Mech. Anal., v. 10, 1962, pp. 171-179. MR 86 «3189.

4. H. F. WeINBERGER, "Lower bounds for higher eigenvalues by finite differenoe mothods," Pacific J. Math., v. 8, 1958, pp. 339-368. MR 21 *6097.

5. B. Wendrorf, Computing the Critical Freguencies of Stepped Shafts, Los Alamos Soien. tific Laboratory Report, LAMS-2954, 1963. 